\title{
PSYCHE.
}

\section{THE INVERTED HYPOPYGIUM OF DASYLLIS AND LAPHRIA.}

BY ROBERT E. SNODGRASS, PULlMAN, WASH.

The Asilid genera Dasyllis and end may be also deeply notched as in Laphria present the curious anomoly of D. grossa (Fig. 4) or it may be but having the hypopygium, i. e., the ninth slightly concave as in D. flavicollis and segment of the male, inverted. Figures I and Io clearly show this condition in $D$. grossa and $D$. flavicollis.

The writer is indebted to Professor J. M. Aldrich, of the University of Idaho, for named material on which the following descriptions are based.

The hypopygium consists of a large ovate structure carried by its larger end on a comparatively narrow neck formed of the seventh and eighth abdominal segments and their intervening membranes (Fig. 10). Within the hypopygium is a large cavity, the genital chamber, opening posteriorly. It has a thick swollen floor on which is carried the intromittent organ, and thin dorsal and lateral walls. The dorsal wall presents a large median notch reaching forward almost to the base of the hypopygium. The lateral walls are similarly, but less deeply, notched.

The lower part of the hypopygium is the ninth tergum (IX t.). It consists of a large convex plate, oval to elongateovate in ventral view, with the smaller posterior end truncate. The posterior

\section{L. vultur.}

The upper and lateral parts of the hypopygium consist of the ninth sternum (IX s.). On account of the dorsal and lateral notches of the hypopygium, the sternum has the form of a basal semicircular plate with two large dorso-lateral lobes projecting backward (Figs. I, 2, 4 and ro, IX s.) Each of these lobes carries, within the genital chamber, two pairs of large movably attached appendages ( $a$ and $b$ ). One pair $(a)$ is lateral and is articulated to the dorsal edge of the lateral notch of the hypopygium (Figs. I, 5 and $10, a$ ). Each of these (Fig. 9) is laterally flattened and strongly curved dorsally, where it ends in one or two heavy claws that project out of the dorsal notch. The other pair (b) is dorsal and is born by two lobes at the anterior angles of the dorsal notch of the hypopygium (Figs. 2 and $5, b$ ). These appendages (Fig. 8) vary considerably in shape. They are generally bent somewhat laterally. In some species they are expanded basally, in others terminally; in some they are prong-like, in 


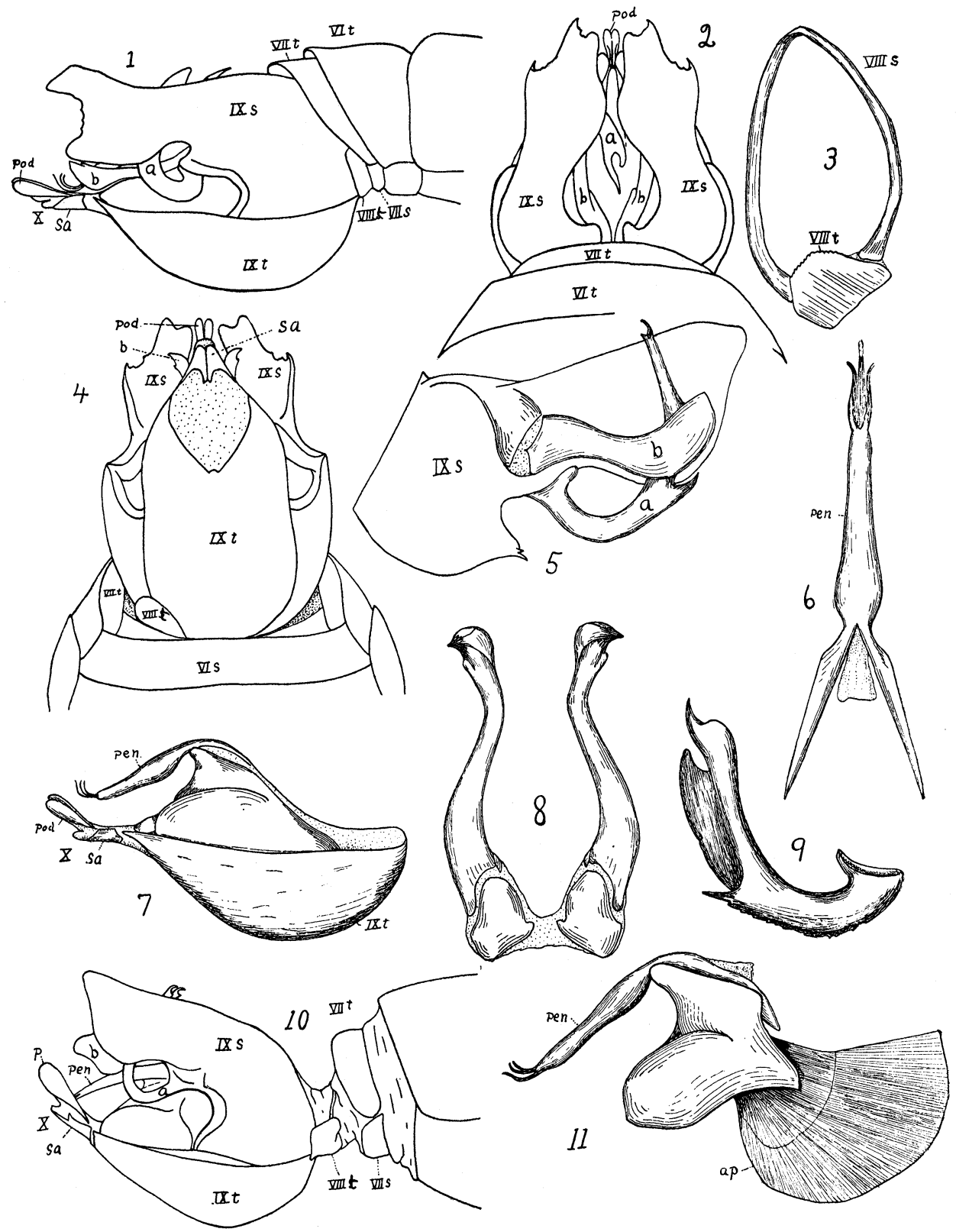

INVERTED HYPOPYGIUM OF DASYLLIS AND LAPHRIA. 
others they are spoon-shaped. In $L$. vultur the tip of each is formed of three plates set at right angles to one another.

The penis (Figs. 6, 7 and $\mathrm{I} I$, pen.) is a chitinous tube terminating in three slender prongs. Apparently the seminal passage divides into three tubes at the bases of these and opens by three apertures at their tips. The penis (Fig. 6) is carried on an elevated support (Fig. 7) on the floor of the genital chamber. The three terminal prongs project posteriorly from the latter over the tenth segment. Attached to the interior of the support is a large muscle apodeme (Fig. $\mathrm{I}$ I, $a p$.).

On account of the inverted position of the ninth segment, the tenth segment (X) comes to lie below the mouth of the genital chamber instead of, as normally, above it. It is composed of the ordinary characteristic parts. There is a divided suranal plate (sa) lying here below the anus, and two elongated podical plates (pod) lying above the anus.

The eighth segment is partially rudimentary. It consists of a narrow curved bar forming the dorsal and lateral parts (Figs. 3 and ro, VIII $s$ ), and of a small plate (Figs. I, 3, 4 and ro, VIII $t$ ) applied to the right lower aspect of the base of the hypopygium. The eighth segment is symmetrical in shape but asymmetrical in position. Normally it is almost hidden within the seventh segment (Fig. I).

The rectum lies in the right side of the body cavity of the hypopygium, turn- ing in mesially at the base of the tenth or anal segment. Hence, the hypopygium must have revolved to the right from above. If this is so, then the small dextro-ventral plate of the eighth segment is probably the eighth tergum which has revolved through only about $150^{\circ}$. The seventh segment is normal, the revolution having taken place entirely back of it. It is small and is mostly concealed within the sixth segment.

\section{Explanation of Plate.}

Fig. I, Dasyllis grossa, lateral view of hypopygium ; Fig. 2, D. grossa, dorsal view of hypopygium; Fig. $3, D$. grossa, eighth segment ; Fig. 4, D. grossa, ventral view of hypopygium; Fig. $5, D$. posticata, interior view of right half of ninth sternum, showing attachments of appendages $a$ and $b$; Fig. 6, D. posticata, dorsal view of penis ; Fig. $7, D$. grossa, lateral view of ninth tergum and penis, and tenth segment; Fig. 8, D. grossa, appendages $b$; Fig. 9, D. grossa, appendage $a$; Fig. $1 \circ, D$. flavicollis, lateral view of hypopygium; Fig. I r, D. Aavicollis, lateral view of penis and its sup port. VI $t$, VII $t$, VIII $t$, IX $t$, sixth to ninth abdominal terga; VI $s$, VII $s$, VIII $s$, IX $s$, sixth to ninth abdominal sterna; X tenth segment; $a$, lateral appendage of ninth sternum; $b$, dorsal appendages of ninth sternum; pen, penis; $a p$, apodeme; $s a$, suranal plate; pod, podical plates. 

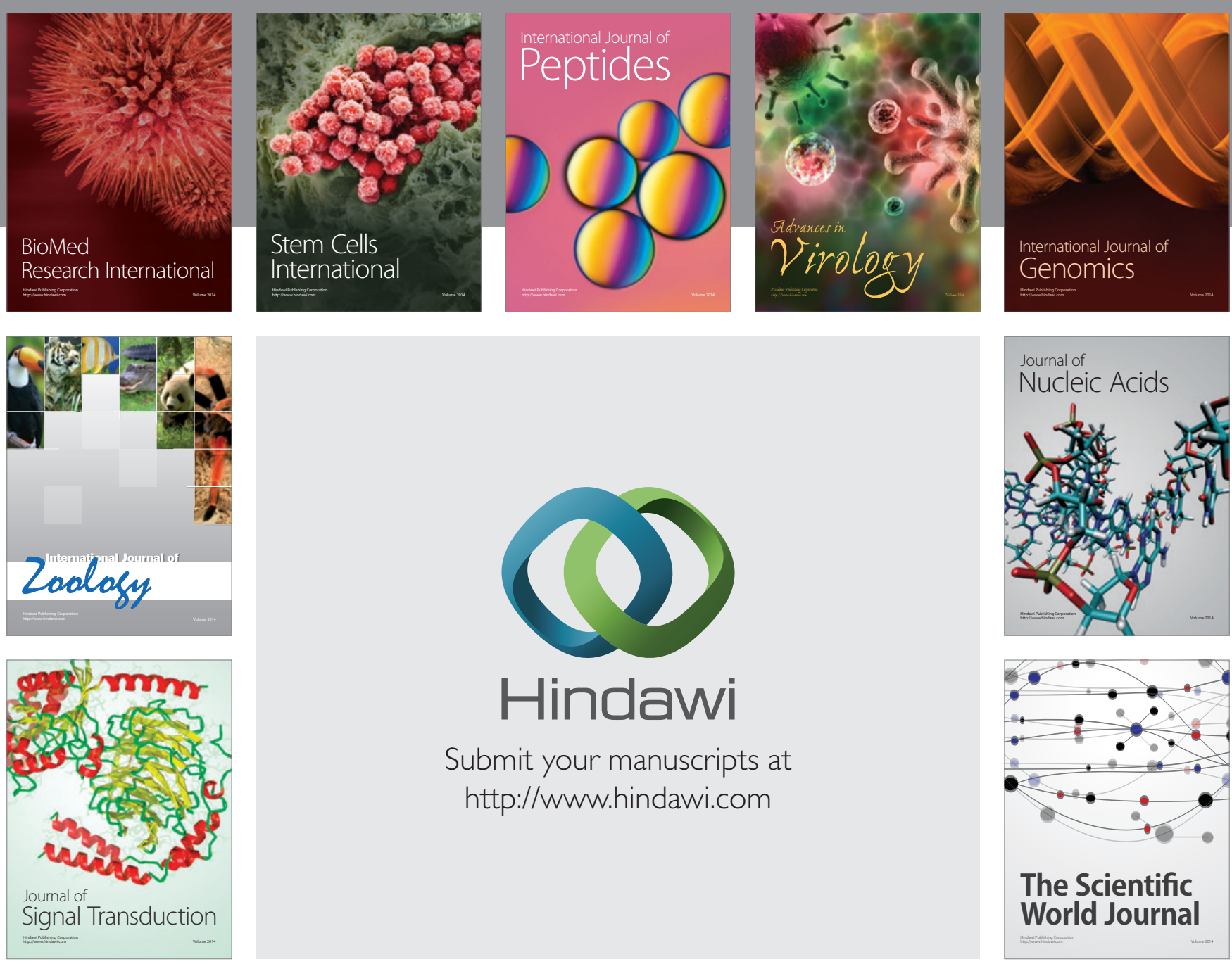

Submit your manuscripts at

http://www.hindawi.com
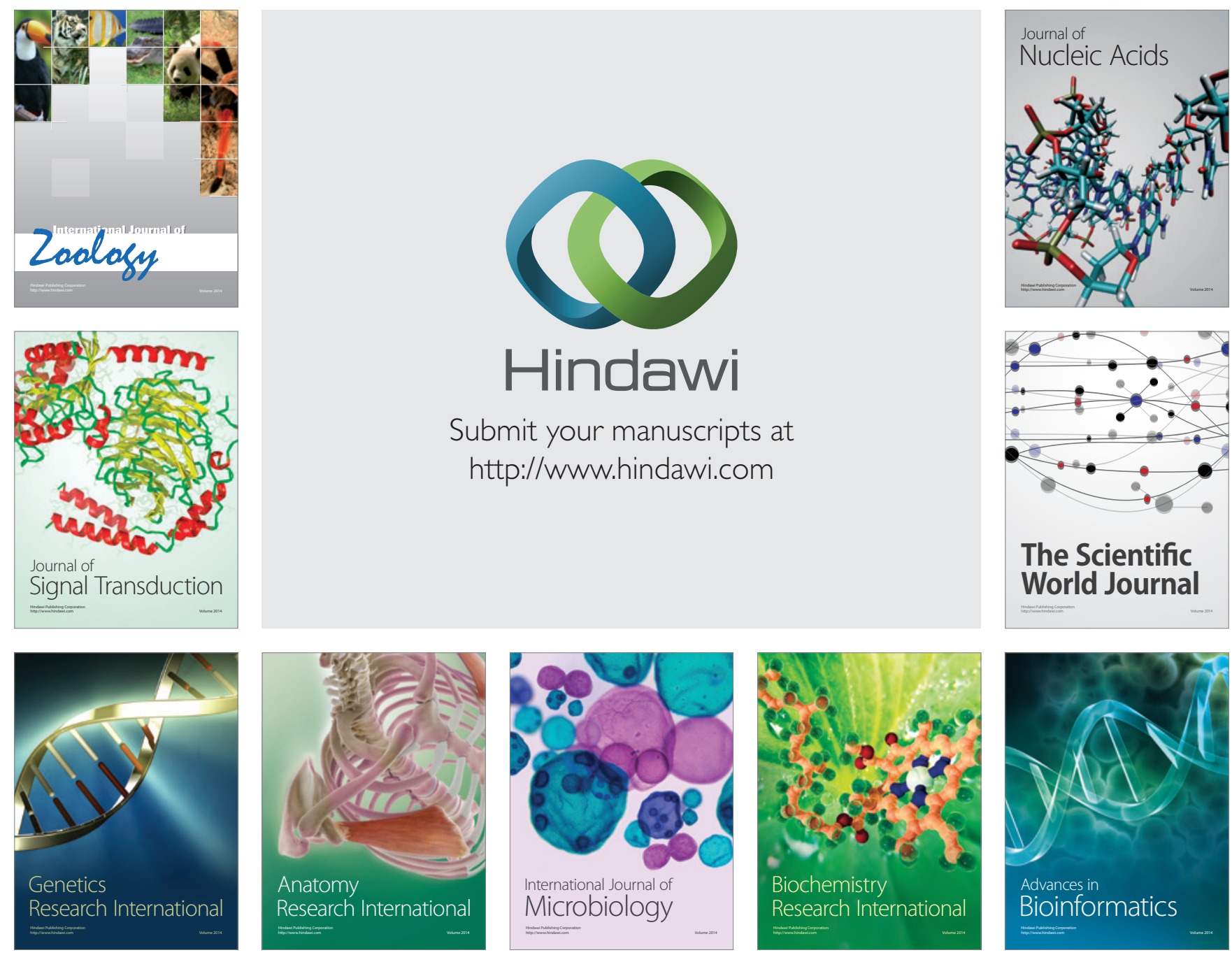

The Scientific World Journal
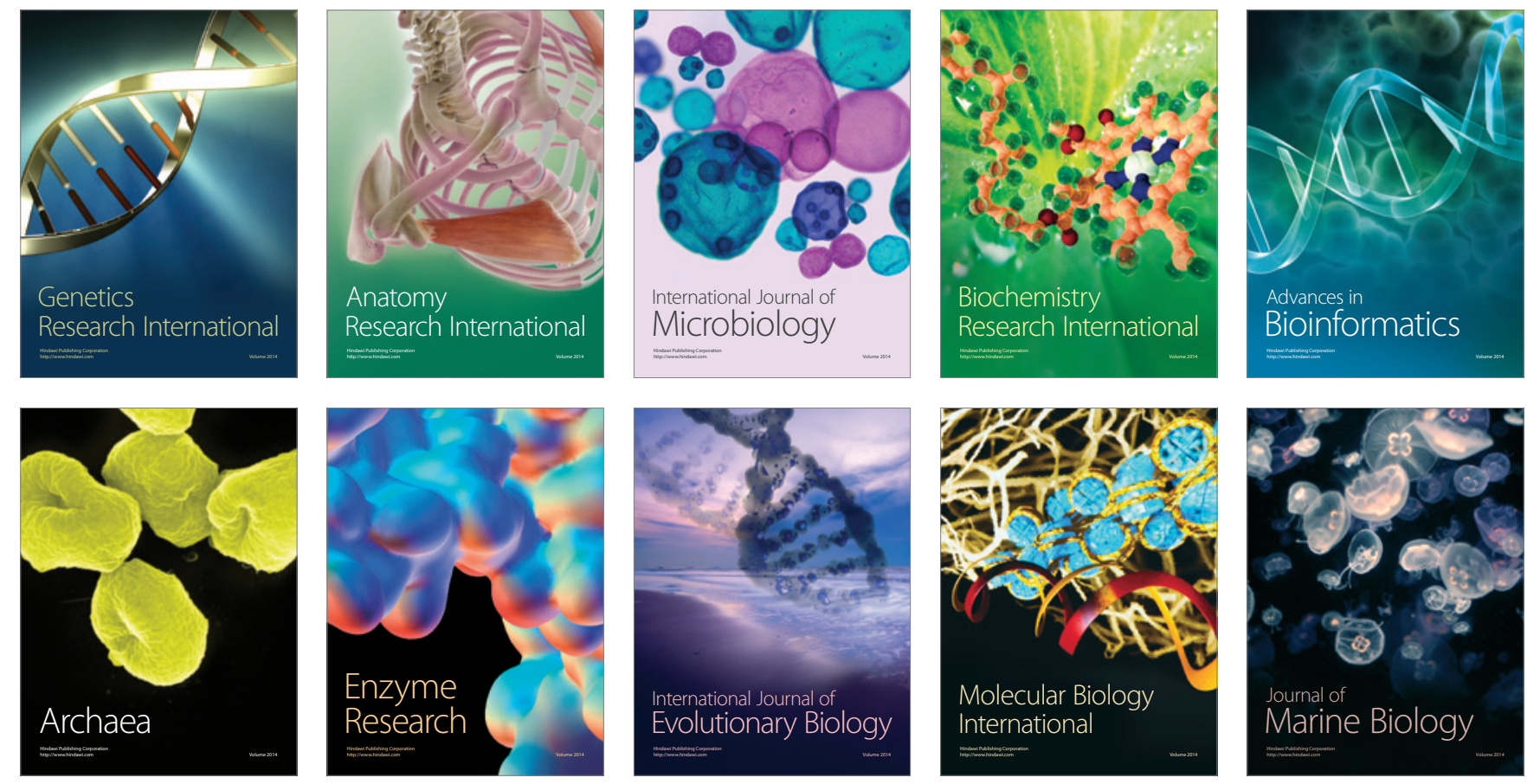